\title{
EDITORIAL
}

\section{Precision Measurement Physics and Its Methodology}

\author{
Chao-hui YE (叶朝辉 $)^{1}$, Jia-ming LI $(\text { 李家明 })^{2,3}$, Jun LUO $(\text { 罗俊 })^{4}$ \\ ${ }^{1}$ Wuhan Institute of Physics and Mathematics, Chinese Academy of Sciences, Wuhan 430071, China \\ ${ }^{2}$ Department of Physics, Tsinghua University, Beijing 100084, China \\ ${ }^{3}$ Department of Physics, Shanghai Jiao Tong University, Shanghai 200030, China \\ ${ }^{4}$ Department of Physics, Huazhong University of Science and Technology, Wuhan 430074, China \\ E-mail: ye@wipm.ac.cn,lijm@sjtu.edu.cn,junluo@mail.hust.edu.cn \\ Received February 18, 2009; accepted February 23, 2009
}

Precision Measurement Physics deals with the frontier problems in science, and plays a multi-disciplinary and fundamental role in strongly advancing the sciences. It is well known that an improvement of measuring precision in physics by an order of magnitude often implies a new or unknown effect to be explored, consequently even a new physical law to be established. There is no doubt that the development of modern physics is so closely related to precision measurements. During the past few decades, the methodologies and physics of precision measurements have achieved tremendous breakthroughs, and therefore have extended our understanding of the physics world. In addition, some technologies developed in this process have been applied in our daily life. Also, we can expect Precision Measurement Physics to make more significant progress toward verifying the basic laws of physics, and to find more practical applications in the future.

The relationship between physics and precision measurements can be viewed from these two aspects: (1) to conduct researches in physics by means of precision measurement techniques; and (2) to develop or to invent new precision measurement techniques by means of state-of-the-art research outcomes in physics. Therefore, the scope of Precision Measurement Physics should include precision measurements of physical constants, experimental verifications of physical laws, and developments of advanced and novel measurement techniques. To achieve higher precision is a common goal for all those scientists involved in fundamental physics or in measurement technologies.

In order to focus on the scientific problems in Precision Measurement Physics and to discuss the development strategy of Precision Measurement Physics in China, the $327^{\text {th }}$ Xiangshan Science Conference was held at Huazhong University of Science and Technology, Wuhan, on July 13-15, 2008. The theme of this meeting was "Precision Measurement Physics and Its Methodology." Forty-five scientists, including local and overseas scholars, attended this meeting, and discussions were held around these following topics: "The trends of precision measurement physics," "Time and frequency standards," "Determinations of fundamental physics constants," "Precision measurements in extreme conditions," "The applications of precision physics measurements," and "Precision measurement physics and the scientific instruments."

The $327^{\text {th }}$ Xiangshan Science Conference was successful and fruitful. As a result of this conference, we, the co-chairmen, asked for the participants' papers at the end of the meeting. The contributions from the attendees have been edited for this special topic of Frontiers of Physics in China. In addition, all the papers went through a standard review process of the journal, and the authors made the necessary revision in view of the reviewing comments. As we put this current topic out, we express our deepest appreciation to the authors for their great efforts, and to the Journal editors for their kind assistance.

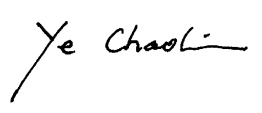

Chao-hui YE

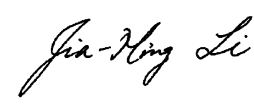

Jia-ming LI

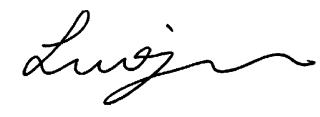

Jun LUO

Academician, CAS Academician, CAS 